\title{
A Conceptual Model for Grid Learning Services Automatic Composition
}

\author{
Gustavo Gutiérrez-Carreón ${ }^{1}$, Thanasis Daradoumis ${ }^{1}$ and Josep Jorba ${ }^{1}$ \\ ${ }^{1}$ Open University of Catalonia, Av. Tibidabo 39-43 - 08035 Barcelona, Spain \\ \{ggutierrezc, adaradoumis, jjorbae\}@uoc.edu
}

Keywords: Learning Grid, Learning Services, Semantic Web.

This work proposes an initial model for the automatic composition of Grid based learning services based on the semantic capabilities and metadata of e-learning frameworks.

There are three principal motivations for Learning Grid Services Composition: build a more powerful service using basic existing services, fulfill service requester's requirement better, and enhance resource reuse while reducing the cost and time of a new service development.

Let us consider a learning Grid as a set of resources and services distributed in a network with the service model based on the IMS abstract framework [1], where learning services can be composed by others allocated in different repositories inside the network. The model we propose for the automatic composition of learning services is based on the use of the defined syntactic and semantic characteristics of the different levels of services involved in the Learning Abstract Framework. The design of the model is presented in the Fig. 1 and is described below.

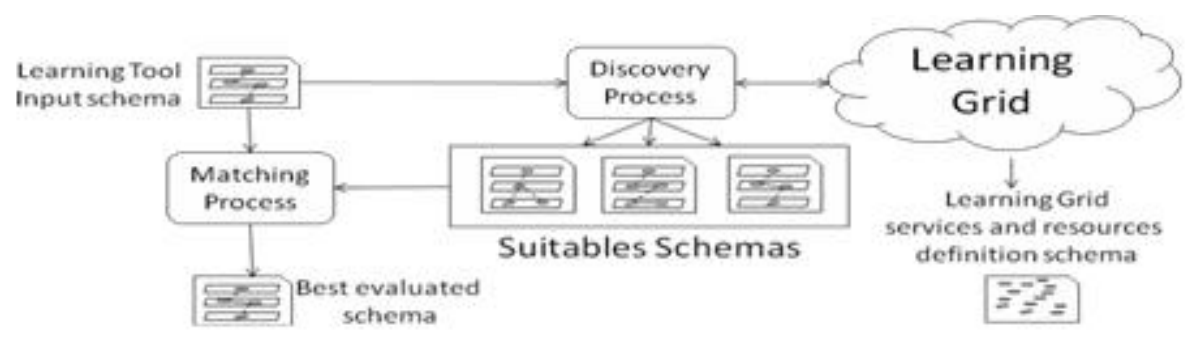

Fig. 1 Grid Learning services automatic composition

Using web languages, such as RDF, DAML+OIL, and OWL, it is possible to create semantically rich data models that are denominated semantic schemas [2]. These semantic schemas are made up of triples (subject-predicate-object), where subjects and objects are entities, and predicates indicate relationships between those entities. Discovery is the process of finding Web services with a given capability [3]. In general, discovery requires that Web services advertise their capabilities with a registry, and that requesting services query the registry for Web services with particular capabilities. In our model, once the semantic schema of the tool or learning service that we want to build is designed, we have to pass it to our discovery process that will locate a set of different level services in the Learning Grid. The operation of these services as a whole allows us to carry out the processes defined in the schema. 
This process consists primarily on comparing inputs and outputs of a service as semantic concepts represented in the schema to incorporate semantics about learning services accessible by a discovery service. The result of the search will be a group of suitable schemas that conforms to the functional process described in our initial schema. Schema and ontology matching aim at identifying semantic correspondences between metadata structures or models such as database schemas, XML message formats, and ontologies. The resulting schemas of discovery process will be compared to the initial schema through a Matching process that is based on a structural matching approach and on a taxonomy matcher and whose result will be the best evaluated schema for our learning tool or services. The taxonomy matcher draws on the given taxonomic Metadata to deduce whether two elements are related semantically. The result of this matching process will be a ranking of semantic matching results. This ranking can be used in conjunction with other user-defined constraints to inform of an exact, or potentially useful web-service capability match.

Comparing our conceptual model with the work presented in [4 and 5], our approach represents a complete alternative solution since, on the one hand, we provide a multi-level learning services composition method that enables the construction of complex learning services by means of other low level services, depending on the nature of the learning abstract framework. On the other hand, our approach takes advantage of the semantic and syntactic characteristics of learning services, which facilitates a totally automatic construction of new learning tools based on others previously created. Future work aims at the full implementation of the conceptual model presented in this work in a Grid environment with a real time composition of learning collaborative scenarios and portals based on the grid

\section{Acknowledgments}

This work has been partially supported by the Spanish Ministry of Education under grant TSI2005-08225-C07-05.

\section{References}

1. IMS Global Learning Consortium, IMS Abstract Framework: White Paper, 2003

2. Paul Mutton , Jennifer Golbeck, Visualization of Semantic Metadata and Ontologies, Computer Science, University of Kent at Canterbury, 2003

3. Jennifer M. Schopf, Mike D'Arcy, Neill Miller, Laura Pearlman, Ian Foster, and Carl Kesselman, Monitoring and Discovery in a Web Services Framework: Functionality and Performance of the Globus Toolkit's MDS4, Argonne National Laboratory Tech Report ANL/MCS-P1248-0405, April 2005

4. Ching-Jung Liao, Fang-Chuan Ou Yang, A Workflow Framework for Pervasive Learning Objects Composition by Employing Grid Services Flow Language. Proceedings of the IEEE International Conference on Advanced Learning Technologies (ICALT'04), pp: 840 841, ISBN:0-7695-2181-9, 2004

5. Shalil Majithia, David W.Walker, W.A.Gray, Automated Composition of Semantic Grid Services, International Conference on Autonomic Computing (ICAC'04), May, 2004 